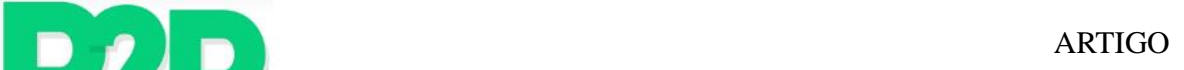

\title{
DEVENIR PACHAMAMA DEL CONOCIMIENTO ${ }^{1}$
}

\author{
Xabier E. Barandiaran \\ IAS-Research Centre for Life, Mind and, Society, Dept. Philosophy, \\ University School of Social Work, UPV/EHU - University of the Basque Country, España \\ DatAnalysis15M Research Network, Cyberspace, Planet Earth \\ IAEN, Instituto de Altos Estudios Nacionales, Quito, Ecuador \\ Daniel Vazquez \\ IAEN, Instituto de Altos Estudios Nacionales, Quito, Ecuador \\ ALabs.Org, España
}

\section{Resumen}

Este el documento madre (ampliable y revisable) para el diseño de un proceso de colaboración y participación social (popular e institucional) y de expertos que culmine en un cumbre productiva (no meramente expositiva). El objetivo es desencadenar y coordinar un proceso participativo a escala global y de inmediata aplicación nacional para el cambio de matriz productiva hacia una sociedad del conocimiento común y abierto en el Ecuador, que resulte en 10 documentos base para el desarrollo legislativo y de políticas de Estado (orientadas al código orgánico para la economía social del conocimiento) así como de utilidad para las redes productivas de conocimiento que ya existen en Ecuador. Se detalla el marco conceptual, económico y filosófico del proceso y del contexto histórico y económicocognitivo, los principios organizativos que regirán el proceso, las herramientas digitales colaborativas y comunicativas y una propuesta de planificación de todo el processo cumbre.

\section{Palabras clave}

Sociedad del conocimiento. FLOK (Free/Libre Open Knowledge) society. Sumak kawsay. Sumak yachay. Pachamama del conocimiento. Investigación colaborativa. Cumbre-proceso. Copyleft. Software libre. Común. Procomún. Autonomía cognitiva. Economía comunitária. Economía social del conocimiento.

\section{TORNANDO-SE PACHAMAMA DO CONHECIMENTO}

\section{Resumo}

Este é o documento mãe (ampliável e revistável) para o desenho de um processo de colaboração e participação social (popular e institucional) e de especialistas que culminou em uma Cúpula produtiva (não meramente expositiva). $O$ objetivo é desencadear e coordenar um processo participativo na escala global e de imediata aplicação nacional para a mudança da matriz produtiva rumo a uma sociedade do conhecimento comum e aberto no Equador, que resulte em 10 documentos base para o desenvolvimento legislativo e de políticas de Estado (orientadas ao código orgânico para a economia social do conhecimento) assim como de utilidade para as redes produtivas do conhecimento que já existem no Equador. Detalha-se o marco conceitual, econômico e filosófico do processo e do contexto histórico e econômico-cognitivo, os princípios organizativos que regerão o processo, as ferramentas digitais colaborativas e comunicativas e uma proposta de planejamento de todo o processo Cúpula.

\section{Palavras-chave}

Sociedade do conhecimento. FLOK (Free/Libre Open Knowledge) society. Sumak kawsay. Sumak yachay. Pachamama do conhecimento. Pesquisa colaborativa. Cúpula-processo. Copyleft. Software livre. Comúm. Procomúm. Autonomia cognitiva. Economia comunitária. Economia social do conhecimento.

\footnotetext{
${ }^{1}$ FLOK Society Design Process+Summit sembrando el sumak yachay (buen conocer), v.1.4.
} 


\begin{abstract}
This is the mother document (expandable and revisable) for the design of a collaborative process and social participation (popular and institutional) and from specialists that culminated in a productive summit (not merely expository). The goal is to initiate and coordinate a participatory process on a global scale and immediate national application for change of the productive matrix towards a society of common knowledge and open in Ecuador, resulting in 10 documents basis for legislative development and state policies (oriented organic code for social knowledge economy) as useful for the production of knowledge networks that already exist in Ecuador. Details conceptual framework, economic and philosophical of the process and the historical, economic and cognitive context, the organizational principles governing the process, collaborative and communicative digital tools and a proposal for planning of the Summit process.
\end{abstract}

\title{
Keywords
}

Knowledge society. FLOK (Free/Libre Open Knowledge) society. Sumak kawsay. Sumak yachay. Pachamama knowledge. Collaborative research. Summit-process. Copyleft. Free Software. Common. Cognitive autonomy. Community economy. Social knowledge economy.

\begin{abstract}
"Como hijos de Pachamama, de la Madre Tierra, ofrecemos al mundo nuestros principios y códigos culturales, espirituales, lingüísticos e históricos, los conocimientos y saberes ancestrales de nuestros abuelos, la memoria histórica que descansa en la arquitectura, en la cerámica, en la textilería, todo el Saber guardado que nuestros ancianas y ancianos susurran en el silencio y que podemos "leer" en sus arrugas y en nuestros libros de piedra, el hablar de los antepasados con que lagos y mares humedecen nuestras lenguas, el acontecer ancestral que nuestros genes despiertan y hablan en nosotros, que los montes y nevados nos dialogan, que los vientos nos soplan en los oídos." CHOQUEHUANCA ${ }^{2}$
\end{abstract}

"La Revolución del Conocimiento, que propone la innovación, la ciencia y la tecnología, como fundamentos para el cambio de la matriz productiva, concebida como una forma distinta de producir y consumir. Esta transición llevará al país de una fase de dependencia de los recursos limitados (finitos) a una de recursos ilimitados (infinitos), como son la ciencia, la tecnología y el conocimiento." SEMPLADES, Plan Nacional del Buen Vivir 2013-2017, p.19

"Ipsa scientia potestas est (el conocimiento mismo es poder, knowledge itself is power)". Francis Bacon, Meditationes Sacrae, 1597.

\section{$1 \quad$ ANTICIPACIÓN}

Es 24 de Febrero 2014, Quito: Sandra (Ecuador, 25 años, Doctora en Trabajo Comunitario) y su pareja Alejandro (Colombiano, 26 años, BA en ingeniería de software, MSc en Global IT Economy) entran en un estadio de baloncesto habilitado para la ocasión. Aún no se lo imaginan pero no van a abandonar ese lugar en los próximos 8 días, un espacio de producción del que ya forman parte desde hace meses, desde que Sandra entro en el espacio virtual para contribuir en

${ }^{2}$ Choquehuanca Céspedes, D. (2010). Hacia una reconstrucción del buen vivir. America Latina en Movimiento, 452, 8-12. 
el desarrollo de líneas estratégicas de desarrollo digital comunitario de su departamento. Entran. Como si fuera un valle de la selva, contemplan el paisaje como un ecosistema de cables, personas, máquinas, luces y procesos. 36 mesas de trabajo redondas con una media de 10 personas cada una, agrupadas las mesas en grupos de tres formando una especie de trébol funcional en una maraña global. Pantallas gigantes muestran el flujo de información que atraviesa el espacio. Personas van y vienen entre tréboles. Una gráfica muestra el progreso en los documentos de trabajo.

Ahora la pantalla 4 muestra las contribuciones aceptadas al documento "Propuesta estratégica para el procomún científico". La pantalla 1 muestra un resumen del workflow global: 12987 líneas de código jurídico, conceptual, y político son ya estables, 4567 esperan en fase beta, el contador acaba de sumar 4 más. Allá abajo, en el bullicio de esa jungla de conocimiento Sandra y Alejandro van a conocer a Michel Bauwens a quién sólo conocían de los cursos obligatorios online (através de la plataforma en desarrollo http://edx.iaen.edu.ec) que todo participante ha tenido que realizar para entrar a la cumbre, con él comprender cómo definir una economía real del p2p basada en procesos abiertos y participativos. Ahora pueden discutir los detalles con él y avanzar el documento "7. Territorialización del trabajo cognitivo". Sandra se pone a hablar con una parlamentaria europea del Partido Pirata, la semana pasada contactaron para ver cómo implantar políticas de privacidad y software libre en los cibercafés rurales (donde ella descubrió con 17 años la wikipedia a través de un icono con una E que asociaba entonces a internet). Se ponen a trabajar y editar los párrafos 34 y 78 del documento madre. Isaac Hacksimov es el editor que contactará con dos revisores antes de dar el visto bueno a la nueva versión de los párrafos que finalmente ensamblarán un documento base para la asamblea nacional. Alejandro hace turno de traducción y documentación del proceso, unos hackers suecos en Telecomix están twitteando la reunión del grupo de cifrado ciudadano y hacen falta proxies de traducción, ahora le toca traducirle uno de los twits a un parlamentario ecuatoriano preocupado por que el cibercrimen "open data, closed identities". Sigue la discusión, sigue la producción... se está escribiendo una nueva tierra. 


\section{MARCO TEÓRICO: LA PACHA MAMA DEL CONOCIMIENTO COMO HABITAT DEL BUEN SABER (SUMAK YACHAY)}

El conocimiento, aquello que una vez fuera sol lejano, luz arrojada como una espada sobre la oscuridad, blandida por una sola mano (la del chamán, el sacerdote, el alquimista, el filósofo) es hoy, gracias a las nuevas tecnologías, madre tierra de luces, ciberespacio, selva de saberes, ecosistema de culturas y cultivos diversos, múltiples, apropiables, accesibles... inagotables.

Los trabajadores del conocimiento, labradoras culturales, cultivadoras, son hoy 1) el sector de mayor crecimiento económico ${ }^{3}$ (con más de 230 millones de trabajadoras cognitiva ${ }^{4}$ ) y además 2) suponen la mayor contribución al crecimiento económico global ${ }^{5}$. El conocimiento es un factor recursivo (exponencial) en el crecimiento y empoderamiento social: a mayor conocimiento mayor capacidad económica pero también mayor capacidad de adquirir conocimiento que a su vez mejora las condiciones de (re)producción de la vida y del conocimiento... Pero además hay un factor que hace que el conocimiento sea especialmente valioso y beneficioso. A diferencia de otros bienes que son escasos y excluyentes (como las materias primas, el suelo o el petróleo) el conocimiento se crea una sola vez y puede beneficiar para siempre a todas las personas y sociedades. Y decimos que "puede beneficiar para siempre y a todas", que puede, porque la historia nos ha dejado un territorio seco, la propiedad intelectual lo cerca "casi" todo. Ejércitos asfaltan las fronteras del conocimiento, patrullan los cercamientos de las tierras fértiles. Nos encontramos, en definitiva, con el antagonismo constituyente que ha definido la revolución ciudadana del buen vivir en el ámbito del conocimiento y sus soportes cibernéticos: una herencia e imposición colonialista global que imprime un individualismo cognitivo basado en el consumo y transacción del saber en la forma de la propiedad intelectual. A esta herencia se enfrentan, por un lado, las tradiciones indígenas originarias del yachay, de (re)producción de saberes comunitarios y, por otro, las nuevas formas de colaboración del procomún digital de la cultura y la ética hacker. Resuenan los principios de reciprocidad (randi-

\footnotetext{
${ }^{3}$ Es el sector que más crece económicamente: frente a la agricultura, extracción de materias primas, ejércitos, etc.

${ }^{4}$ Manyika, J., Chui, M., Bughin, J., Dobbs, R., Bisson, P., \& Marrs, A. (2013). Disruptive technologies: Advances that will transform life, business, and the global economy. McKinsey Global Institute.

${ }^{5}$ Un país no es más rico porque tenga más petróleo o más fuerza de trabajo, sino porque tiene mayor producción cognitiva: mayor eficiencia en los procesos logísticos, mejores diseños, más información, etc.
} 
randi) y organización del trabajo comunitario ${ }^{6}$ (maki-maki) en lo que podríamos llamar una Pacha Mama digital del conocimiento (el commons del general intellect).

Figura 1: Triada conceptual del proceso-cumbre

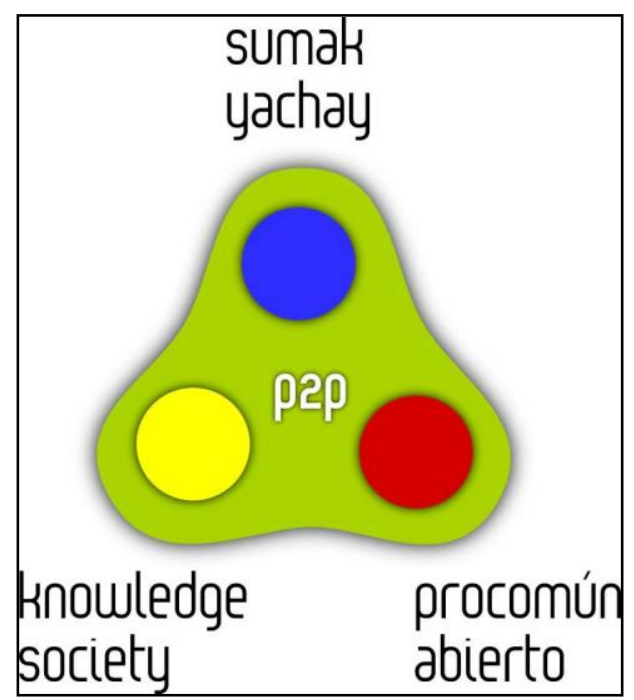

Figura 1: El sumak yachay (buen conocer) arriba, simboliza la aspiración a la armonía con la pachamama del conocimiento. Knowledge society abajo a la izquierda hace referencia a la sociedad del conocimiento global como hábitat de los procesos de cambio productivo en Ecuador. El procomún abierto declina la nueva matriz productiva y sus valores de lo común como fundamento de riqueza colectiva y lo abierto como condición del desarrollo colaborativo del empoderamiento social.

Frente a los cercamientos de los territorios $\operatorname{cognitivos}^{7}$ se abre la posibilidad real de la siembra, el cuidado y fertilización de las tierras comunales del conocimiento aberto ${ }^{8}$. El gobierno del Ecuador tiene la firme voluntad de promover, preservar e incentivar una sociedad del conocimiento libre, una economía del procomún cognitivo, una Pacha Mama de la cultura, la tecnología, los saberes y la información libre, abierta, accesible, de todos. Toda forma de vida implica una forma de conocimiento, de acoplamiento con el medio, de regulación del intercambio de materia y energía 9 . Habitar es conocer y el buen vivir requiere un buen saber. El conocimiento abierto, común, compartido es un habitat para el buen vivir, una necesidad. Sólo el mejor vivir exige

\footnotetext{
${ }^{6}$ Makas, L. (2010). Sumak Kawsay: la vida en plenitud. America Latina en Movimiento, 452, $14-17$.

${ }^{7}$ Murdock, G. (2001). Against enclosure: Rethinking the cultural commons. In British Cultural Studies (pp. 443-460).

${ }^{8}$ Bauwens, M., Mendoza, N., \& Iacomella, F. (2012). Synthetic Overview of the Collaborative Economy. P2P Foundation. Tomado de: http://p2p.coop/files/reports/collaborative-economy-2012.pdf

${ }^{9}$ Bourgine, P., \& Stewart, J. (2004). Autopoiesis and cognition. Artificial life, 10(3), 327-345.

Varela, F. J. (1997). Patterns of life: Intertwining identity and cognition. Brain and cognition, 34(1), $72-87$.
} 
excluir al otro del acceso al conocimiento, el buen vivir, sumak kawsay, demanda, en esta globalidad del conocimiento de un sumak yachay de los saberes (nuevos y viejos). Es por tanto necesario desarrollar el buen conocer, aquél que beneficia a todos, que crea un entorno rico y fértil para la vida cultural, social, económica, política (ver Figura 1 en la página anteior). En definitiva, crear una matriz productiva basada en el conocimiento común y abierto.

Ya existen pequeños y grandes jardines del buen conocer, incluso algunos bosques pero... ¿Cómo se siembra una selva amazónica, un ecosistema completo de conocimiento libre? Un ecosistema que no sea fácilmente desertizable, ni atacado por especies invasivas que agoten los recursos colectivos, que no sea cruelmente expoliada. ¿Cómo? Traer las cinco flores más bellas del mundo no va a servir, lanzar 3 millones de semillas en territorio desértico tampoco, ni valdrá diseñar un jardín francés que requiera un ejército permanente de jardineros para mantener rectos sus laberínticos arbustos. La naturaleza misma tiene la clave: la riqueza ecosistémica y la armonía homeodinámica de una selva no se la da la latitud ni la cantidad de sol que recibe su territorio, ni los ríos que lo atraviesan. La misma cantidad de luz cae sobre un desierto que sobre un Amazonas, la misma cantidad de agua cae sobre el mar que sobre el Yasuní. La clave de una selva es que es tan poderosa y rica que crea y regula su propio clima ${ }^{10}$, creando sus ríos, sus sombras, su humus, su tierra.

Tenemos que empezar a sembrar, traer plantas, cultivar cerca de los oasis, apartar las barreras, y comenzar a vivir de la y en la selva según crece en nuestras manos, permitir que se conecten los bosques, abrir los jardines, levantar el asfalto, dejar entrar el sol y la lluvia, permitir que la tierra respire, limpiar el agua, alejar la industria tóxica, prevenir los monocultivos... y la selva se extenderá.

\section{MARCO POLÍTICO-INSTITUCIONAL EN ECUADOR Y EL MOVIMIEN- TO DEL SOFTWARE LIBRE Y EL COPYLEFT}

El cambio de matriz productiva hacia una sociedad del conocimiento común y abierto es una de las apuestas centrales del nuevo Plan Nacional del Buen Vivir de Ecuador:

"La Revolución del Conocimiento, que propone la innovación, la ciencia y la tecnología, como fundamentos para el cambio de la matriz productiva, concebida

\footnotetext{
${ }^{10}$ Lovelock, J. (2000). Gaia: A new look at life on earth. Oxford University Press. Lovelock, J. E., \& Margulis, L. (1974). Atmospheric homeostasis by and for the biosphere: the Gaia hypothesis. Tellus, 26(1-2), 2-10.
} 
como una forma distinta de producir y consumir. Esta transición llevará al país de una fase de dependencia de los recursos limitados (finitos) a una de recursos ilimitados (infinitos), como son la ciencia, la tecnología y el conocimiento." (Plan Nacional del Buen Vivir 2013-2017, p.19)

Esta tarea de transformación, sin embargo, es extraordinariamente compleja, en una matriz productiva basada en el conocimiento convergen aspectos tan diversos como las políticas de desarrollo tecnológico, las instituciones académicas, la gestión de los saberes ancestrales, los formatos de documentación y datos públicos, las regulación del sector de telecomunicaciones o el desarrollo de software y la gestión de derechos culturales. El éxito de esta matriz productiva radica precisamente en un diseño integrado de toda una serie de transformaciones a diferentes escalas y en diferentes áreas de la producción, de la acción social y de los procesos institucionales. Además, el gobierno de Ecuador no apuesta por cualquier tipo de economía del conocimiento, sino por un conocimiento común y abierto:

La estrategia de acumulación, distribución y redistribución, en concordancia con el Programa de Gobierno 2013-2017, propone una gestión del "conocimiento común y abierto". Este modelo de gestión incluye la generación de ideas creativas, su aprovechamiento, la potencial producción de nuevos bienes y servicios y la distribución de sus beneficios. La gestión del conocimiento - visto como un bien público, común y abierto- expresa un principio según el mandato constitucional y es economicamente más eficiente que los modelos cerrados. (Plan Nacional del Buen Vivir 2013-2017, p.67).

En esta apuesta convergen dos grandes proyectos políticos: el del buen vivir de la revolución ciudadana de Ecuador, por un lado, y el del movimiento del software y cultura libre por otro. El primero recupera el conepto de sumak yachay de la cultura indígena andina como eje programático para una revolución ciudadana: definir y desarrollar una transformación social basada en el equilibrio con la naturaleza y las relaciones humanas que permite el florecimiento personal sin excluir ni someter a la naturaleza o al ser humano ${ }^{11}$. El segundo gran proyecto político surge de la cultura hacker y del movimiento copyleft, el p2p y la defensa del procomún en la era tecnológica: la democratización del conocimiento y la técnica a través de las redes electrónicas, el empoderamiento social a través de los ilimitados recursos del conocimiento y la técnica e impul-

\footnotetext{
${ }^{11}$ Acosta, A. (2010). El Buen Vivir en el camino del post-desarrollo: Una lectura desde la Constitución de Montecristi. Policy Paper, 9. Asamblea Constituyente. (2008). Constitución de la República del Ecuador. Ciudad Alfaro. Gudynas, E. (2011). Buen Vivir: Germinando alternativas al desarrollo. América Latina en movimiento, 462, 1-20. Houtart, F. (2011). El concepto de Sumak kawsay (buen vivir) y su correspondencia con el bien común de la humanidad. Revista de filosofía, 29(69), 7-33. SEMPLADES. (2013). Plan Nacional para el Buen Vivir 2013-2017. Quito, Ecuador.
} 
sados por la cooperación socio-técnica distribuida. Los logros y servicios que la comunidad internacional del software libre han generado en los últimos 20 años son el mejor ejemplo de gestión del conocimiento y de una economía productiva del buen vivir. Así lo demuestra GNU/Linux, un sistema operativo libre y completo que cubre las necesidades de individuos e instituciones haciendo frente en calidad y cantidad a las ofertas equivalentes basadas en el software privativo. El precio estimado de una distribución de GNU/Linux como Debian ya en el año 2001 rondaba los 1,848,225,000 USD ${ }^{12}$, en 2007 la cantidad de código incluida en la distribución había se había multiplicado ya por $6^{13}$, hoy día podríamos estimar a la baja que el coste teórico de producción de GNU/Linux es de más de 10,000 millones de USD.

Junto al software libre han surgido un gran número de movimientos que luchan con el mismo objetivo emancipador, colaborativo y abierto en áreas que van desde el diseño industrial hasta las biotecnologías, pasando por la cultura libre, la defensa de los derechos digitales en la red o el libre acceso al conocimiento científico, buscando una economía distribuida del conocimiento. Estos movimientos se unen a las luchas comunitarias que intentan rescatar y proteger los sabe-res ancestrales así como los recursos naturales del expolio y monopolios biotecnológicos ${ }^{14} \mathrm{y}$ culturales de las grandes industrias de patentes biológicas y del entretenimiento cultural. En todas estas re-aperturas de lo común se han ensayado tipos de economía sin la forma de la propiedad intelectual y que sobreviven en un contexto de capitalismo cognitivo: editoriales científicas abiertas (open access) como $\operatorname{PLoS}^{15}$ o Frontiersin ${ }^{16}$, pasando por los bancos de semillas y las cooperativas agrícolas ${ }^{17}$ hasta las redes de conectividad libres como Guifi ${ }^{18}$ (con más de 50.000 nodos) o el desarrollo de hardware libre de Arduino ${ }^{19}$ o el diseño abierto y colaborativo para máquinas de construcción y agricultura a menor precio que las ofertas de mercado ${ }^{20}$; por mencionar sólo algunas de las formas de producción, económicamente viables sin financiación pública que están

\footnotetext{
${ }^{12}$ González-Barahona, J. M., Perez, M. O., de las Heras Quirós, P., González, J. C., \& Olivera, V. M. (2001). Counting potatoes: the size of Debian 2.2. Upgrade Magazine, 2(6), 60-66.

${ }^{13}$ Gonzalez-Barahona, J. M., Robles, G., Michlmayr, M., Amor, J. J., \& German, D. M. (2009). Macro-level software evolution: a case study of a large software compilation. Empirical Software Engineering, 14(3), 262-285. doi:10.1007/s10664-008-9100-x

${ }^{14}$ Birke, L. I. A., \& Hubbard, R. (1995). Reinventing biology: respect for life and the creation of knowledge. Bloomington: Indiana University Press. Shiva, V. (2001). Patents: myths and reality. New Delhi; New York, NY: Penguin Books.

${ }^{15}$ http://www.plos.org

${ }^{16} \mathrm{http}: / /$ frontiersin.org

${ }^{17} \mathrm{http}: / /$ beejbachaoandolan.org

${ }^{18}$ http://guifi.net

${ }^{19} \mathrm{http}: / /$ arduino.cc

${ }^{20}$ http://opensourceecology.org
} 
siendo desarrolladas a lo largo y ancho del planeta.

A todo esto se suma la serie de transformaciones ya en marcha que se están dando en Ecuador en los ámbitos de la comunicación, la educación superior o el apoyo internacional a los hacktivistas que luchan por la transparencia y los derechos digitales. Mediante esta iniciativa del devenir Pachamama del Conocimiento, el Ecuador debe establecer un diálogo con la sociedad red, un diálogo productivo e innovador que permita afrontar el reto de pensar los modelos económicos y sociales centrados en lo común, en lo abierto, en esa segunda naturaleza inagotable del ser humano que es el conocimiento.

\section{PRINCIPIOS ORGANIZATIVOS Y BLOQUES TEMÁTICOS}

El reto que se plantea es, como decíamos, extremadamente complejo. Requiere integrar expertos internacionales, investigadoras académicas, hackers, juristas, líderes de comunidades, activistas, empresarios, etc., en un proceso de investigación que permita definir y concretar políticas y principios reguladores que aseguren el éxito de un modelo de sociedade productiva del conocimiento abierto y común (ver Figura 2 en la página 9 para un diagrama de las áreas de desarrollo y los componentes añadidos).

\subsection{Principios organizativos}

Introducimos a continuación una serie de principios organizativos para la arquitectura del proceso así como los bloques temáticos.

- Conceptualizar y procedimentalizar: Hay que trabajar desde el principio (desde este documento mismo) sobre el concepto de proceso abierto, no hablemos de evento o congreso (que es un momento, una foto, un instante del proceso), no hablemos de un gran documento (que es un resultado). Hablemos, hablamos ya, de proceso, de crecimiento, desarrollo, devenir de la Pachamama del Conocimiento. Un proceso modelo, replicable, replicante, ejemplar, fertilizador y territorializado. Abierto y en red, el proceso mismo, no sólo sus resultados o cumbres. Y sobre todo hay que asentar las bases de un marco conceptual de lo que significa un proceso constituyente del sumak yachay y sus resonancias con la cibercultura del procomún. 
- Pensar, investigar, aprender: No habrá cambio de matriz productiva efectiva si no conseguimos ir más allá del conocimiento disponible actualmente. Este proceso requiere desplegar una red de investigación global y local que haga posible un diseño efectivo y operativo de la nueva matriz. Al mismo tiempo los agentes implicados vamos a tener que aprender haciendo en este mismo proceso de investigación-producción.

- Comunicar, seducir, invitar: Necesitamos un grupo de comunicación fuerte y con al menos dos vertientes: una social y otra técnica. Hay que crear una narrativa nacional alrededor de este tema, con un lenguaje directo y potente. Otra narrativa atractiva para el mundo técnico, centrada en el reto que supone investigar y diseñar un proceso de sociedad del conocimiento abierto, algo que movilice a hackers, investigadoras, pensadores, desarrolladores, juristas, etc.

- Conectar: Tenemos que conectar el proceso para un crecimiento sostenible. No basta con conectar a los hubs (los nodos centrales del desarrollo nacional: ministros, altas funcionarias, etc.), éstos ya están hiperconectados y saturados, no hay que conectar (sólo) a lo broadcasting (e.d. uno-a-muchos, micrófono-masas, streaming), tampoco hay que conectar aleatoriamente al mayor número de gente. Hay que conecta libre de escala ${ }^{21}$, a la estudiante de telecomunicaciones con John Perry Barlow, al webmaster de la asociación de linuxeros con el artista del CAC, a Michel Bauwens con la jurista intrépida, a Stallman con la docente del IAEN... de una forma artesana y orgánica. Que conecten todas las escalas a través de conexiones improbables, enlaces de largo alcance, vínculos multiescala. Hay que dejar una nación inyectada, sembrada y regada de procesos de crecimiento cognitivo abierto a todos los niveles fomentando el desarrollo autónomo local con una retroalimentación a escalas estatal, regional y global.

${ }^{21}$ Barabasi, A.-L. (2003). Linked: How Everything Is Connected to Everything Else and What It Means. Plume. Borge-Holthoefer, J., Rivero, A., García, I., Cauhé, E., Ferrer, A., Ferrer, D., Moreno, Y. (2011). Structural and Dynamical Patterns on Online Social Networks: The Spanish May 15th Movement as a Case Study. PLoS ONE, 6(8), e23883. doi:10.1371/journal.pone.0023883 
Figura 2: Arquitectura de la participación en la cumbre

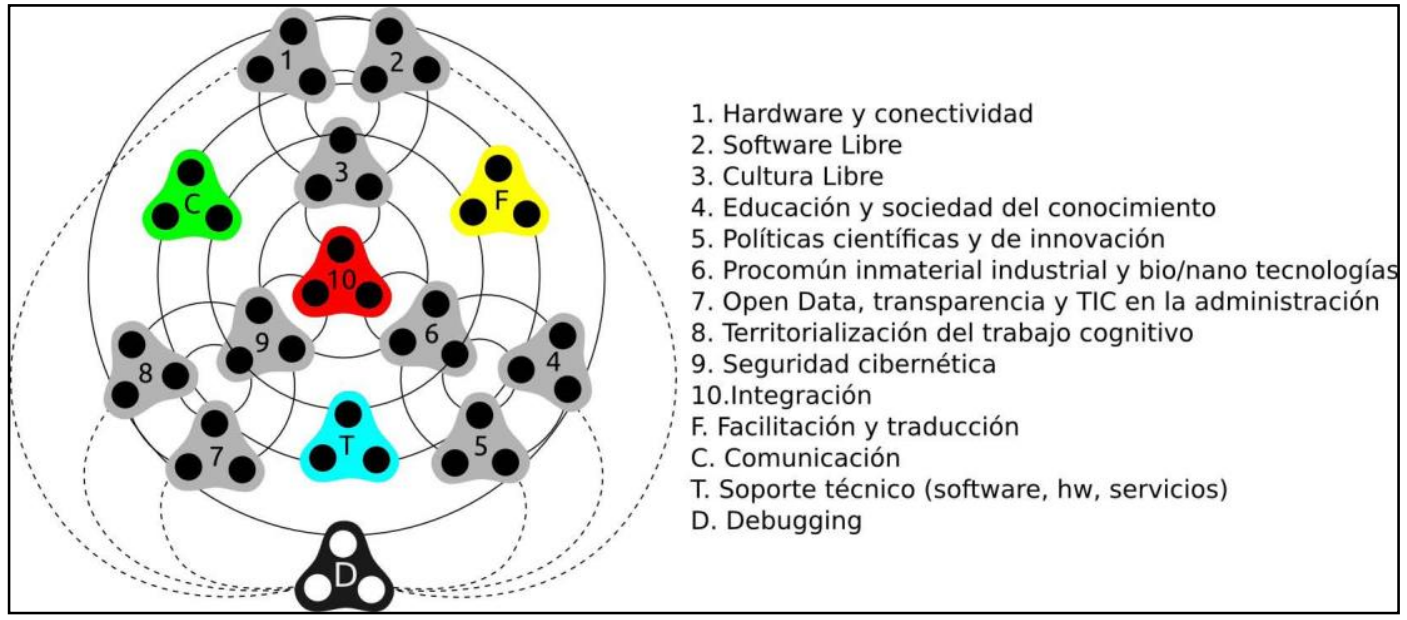

Figura 2: Arquitectura de la participación en la cumbre, cada uno de las áreas está representada por uma triada, las triadas se agrupan a su vez en triadas de áreas de investigación-producción que conectan con una centro integrador. Enlaces transversales entre áreas aseguran una integración distribuida. Equipos transversales de Comunicación, Facilitación, ayuda Técnica y equipo de Debugging que trataría de buscar errores de aplicación, incompatibilidades, y formas de bloqueo de las propuestas para su implantación efectiva.

- Producir: el propio proceso de producción debe sedimentar arquitectura de la participación, que cuando llegue el evento cumbre, la producción crezca sobre el trabajo previo, en los dispositivos inacabados (documentos de trabajo, plataformas de producción, modelos, recursos online, discusiones en proceso...). Podemos pensar en el proceso de producción dividido en sectores o dimensiones de la sociedad del conocimiento que culminen en una serie de documentos programáticos: mapas de redes vitales para el ecosistema tecno-cultural ecuatoriano, análisis de necesidades, potencialidades de crecimiento, identificación de sectores y opciones estratégicas, diseño de modelos de producción, etc.

- Igualdad e integración multicultural: Uno de los factores que no podemos de-jar atrás es a los sectores más desfavorecidos de la sociedad, indígenas, mujeres y afroamericanas deberán de ser preferentemente invitadas. Comenzamos por el uso de un lenguaje no sexista, haciendo uso del genérico femenino para denominar a las personas y buscaremos siempre priorizar el encuentro y la participación de los sectores más desfavorecidos.

- Continuación, cuidado y monitorización: Dar continuidad a los resultados, tanto por áreas como en su implantación social, legislativa e institucional y su reproducibilidad 
internacional en el marco de América Latina. Para ello es fundamental que la participación ciudadana ecuatoriana y global monitorice el desarrollo post-cumbre del desarrollo e implantación de las políticas diseñadas durante el proceso.

- Prototipar producción de conocimiento común y abierto: El evento mismo debe de servir de modelo de producción de conocimiento: el software, la identidad gráfica, los textos, la economía, la contratación, la autoría y los procesos de todo tipo deben estar accesibles y visibles, con licencias copyleft, garantizando una transparencia y auditoría pública ciudadana, así como una reutilización y adaptación a otros FALTA.

\section{2 Áreas temáticas:}

1) Hardware y conectividad: sostenibilidad y soberanía de la infraestructura tecnológicas.

a) Equipos: Hardware libre, sostenible, criterios de compra, problemas del mercado tecnológico y soberanía de hardware, reciclado, etc.

b) Redes: habría que distinguir entre internas y externas, es decir, conectividad institucional/comunitaria/ciudadana y conectividad global (puntos neutros, BGP, anillo sur de UNASUR, cables transoceánicos, satélites, etc.), incluyendo también experiencias de redes libres (ver http://guifi.net)

c) Energía: sistemas de auto-gestión energética para zonas rurales y data-centers etc.

\section{2) Software Libre}

a) Educación: empezar por la base, colegios, telecentros y cibercafés.

b) Implementación: programas de migración de la administración pública, facilitar la migración a empresas, etc.

c) Producción y desarrollo: obligatoriedad de colgar en repositorios públicos los desarrollos hechos a medida para la administración pública. 
d) Políticas de soberanía y autonomía: p.e. obligatoriedad de poder comprar los ordenadores sin sistema operativo, osea sin Windows y con el debido descuento respecto a la opción con Windows.

\section{3) Cultura Libre}

a) Licencias y sociedades de gestión

b) Sostenibilidad de autores: remuneración, fomento, políticas de inversión pública, etc.

c) Medios de publicación: repositorios, etc.

d) Ámbitos: resolver problemas de los puntos anteriores por cada ámbito de proᄀducción (cine, música, literatura, arte, etc.)

\section{4) Educación y sociedad del conocimiento:}

a) Insertar tareas, dispositivos e indicadores de beneficio social del conocimiento en la planificación docente.

b) Copyleft en libros de texto y desarrollo curricular colaborativo y diverso a escala estatal.

c) Gestión, facilitación y certificación de la educación no-formal y saberes no-académicos.

\section{5) Políticas científicas}

a) Ajuste de indicadores y sistemas de evaluación de resultados y procesos científi $\neg$ cos orientados hacia la matriz productiva del conocimiento común y abierto.

b) Open Access y Open Data en ciencia: Publicación y repositorios de resultados y procesos de investigación, repensar toda la comunicación científica.

c) Desarrollo de sistemas de producción colaborativa científica a escala nacional.

d) Arquitectura del sistema de investigación: grupos y proyectos de investigación, redes, agencias de evaluación, eventos e instituciones, etc. 


\section{6) Procomún inmaterial industrial y bio/nano tecnologías}

a) Alternativas a patentes industriales: hacia un open design para la industria local y la autogestión.

b) Derechos de la Pacha Mama y patrimonio natural: defensa contra el expolio de las riquezas genéticas y biotecnológicas de la naturaleza ${ }^{22}$.

c) Salud e industria farmacéutica.

d) Gestión del riesgo y otros problemas asociados

\section{Territorialización del trabajo cognitivo}

a) Economía local del conocimientos: conocimiento e infraestructura desde y para las comunidades.

b) Urbanismo y sociedad del conocimiento.

c) Modelos de espacios de trabajo cognitivo (coworking, cybercafés, cooperativas cognitarias, etc.).

\section{Open Data, transparencia y TIC en la administración pública}

a) Accesibilidad y formatos abiertos de la producción pública

b) Estructura de datos de la administración pública: open data

c) Modelos de gestión y gobernanza digital

\section{Seguridad cibernética}

a) Soberanía tecnológica y programas de ciberdefensa y seguridad nacional.

b) Empoderamiento social en ciberseguridad

c) Certificados y administración pública

${ }^{22}$ Según se recogen en el artículo 322 y 402 de la Constitución de 2008 de la República del Ecuador. 


\section{$5 \quad$ PROPUESTA PLANIFICACIÓN ESTRATÉGICA PARA EL PROCESO}

El objetivo del proceso-cumbre es articular y facilitar un proceso global de investigación y diseño de matriz productiva del conocimiento común y abierto. Para ello es preciso implicar a actores institucionales, regionales, nacionales, y comunitarios. La forma que tomará todo el proceso-cumbre es una serie de documentos abiertos en internet (en forma de wiki) y una red de reuniones de trabajo extendidas en el tiempo y en el (ciber)espacio y convergentes en la cumbre. El equipo de coordinación Devenir Pachamama del Conocimiento (ver sección de recursos humanos más abajo) se encargaría de coordinar la elaboración de los 10 documentos y coordinar el proceso político desde el IAEN en reuniones e informes al Ministerio de Conocimiento y Talento Humano, a la SENESCYT y a otras posibles instituciones públicas ecuatorianas. Se trata de un proceso de investigación, definición, producción y comunicación de políticas públicas que requiere de una arquitectura de la participación que permita ensamblar propuestas y saberes diversos. Uno de los objetivos más importantes es que los 10 documentos resultantes puedan ser de utilidad directa para el código orgánico para la economía social del conocimiento y el plan nacional.

El proceso-cumbre tiene varias fases (ver calendario más abajo): primero se lanza el evento a la participación nacional e internacional. A nivel nacional se contactará con asociaciones e instituciones locales para hacer una serie de reuniones y presentaciones del proyecto (incluyendo la difusión de este documento). A nivel internacional se lanzará una campaña de comunicación en inglés y castellano desde la web y los medios sociales. Se busca la implicación de investigadoras y actores de todo tipo en cada una de las áreas. Para la cumbre final se busca invitar a las/los participantes más activos, financiando el viaje y una remuneración por la semana de trabajo durante la cumbre. Algunas/os de las/los invitadas/os especiales serán contactados y contratados con meses de anterioridad a la cumbre para que contribuyan también durante el proceso, sean coordinadoras/es de área o para que contribuyan con documentos base.

A continuación exponemos los lineamientos de nuestra arquitectura de la participación, las herramientas digitales, el calendario de actividades y resultados y las necesidades de recursos humanos para la realización del proceso-cumbre. 


\subsection{Arquitectura y procesos de participación}

La participación integrará los siguientes procesos:

1. Reuniones de trabajo presencial con asociaciones e instituciones públicas en las que se presentará el proceso-cumbre y se abrirán vías de participación.

2. Encuestas a agentes sociales y expertas internacionales. Estudios sociológicos de las encuestas y mapeo de las fuerzas productivas cognitivas existentes en Ecuador así como sus posibles sinergias con sectores y redes globales.

3. Invitación a expertas nacionales e internacionales para que desarrollen propuestas concretas por cada área que sirvan como borrador.

4. Convocatoria de sesiones de trabajo online en los Etherpad disponibles (ver sección de herramientas) y por salas de voz a través del Mumble.

5. Revisión de las propuestas por expertas y por otros agentes sociales e institucionales.

6. Publicación de textos de trabajo en un wiki (ver herramientas de trabajo) y revisión y reedición colaborativa (mediada por editores/as)

Reuniones de discusión de cada una de las propuestas en su fase media de elaboración y concreción.

7. Reuniones de discusión de cada una de las propuestas en su fase media de elaboración y concreción.

8. Elaboración final en la cumbre.

Esta propuesta de participación y edición colaborativa aún requiere un desarrollo detallado, algunos momentos y procesos del desarrollo están ya incluidos en el calendario provisional pero los resultados mismos de los procesos 1 y 2 irán marcando el camino y los ritmos para los siguientes pasos. Los documentos se elaborarán por áreas temáticas (ver más arriba), cada una de las cuales también es predecible que requiera de ritmos y ajustes propios.

\subsection{Lista preliminar e incompleta de actores a los que podríamos invitar}

A continuación una lista preliminar e incompleta de instituciones y agentes sociales nacionales e internacionales que podrían ser invitados al proceso-cumbre para tomar parte activa en la misma. Esta lista deberá ser completada en las primeras fases de desarrollo. 
Quadro 1: Lista de instituciones y agentes sociales nacionales e internacionales

\begin{tabular}{|c|c|c|}
\hline Internacionales & Sociedad civil & Instituciones ecuatorianas \\
\hline http://p2pfoundation.net & ASLE & SENESCYT \\
\hline $\begin{array}{l}\text { Electronic Frontier Founda- } \\
\text { tion }\end{array}$ & http://creativecommons.ec & $\begin{array}{l}\text { Ministerio Coordinador de Co- } \\
\text { nocimiento y Talento Humano }\end{array}$ \\
\hline http://freeknowledge.eu & Infodesarrollo & SEMPLADES \\
\hline $\begin{array}{l}\text { http://fsf.org Free Software } \\
\text { Foundation }\end{array}$ & Radialistas & $\begin{array}{l}\text { Vicepresidencia (departamento } \\
\text { de cambio de matriz productiva) }\end{array}$ \\
\hline $\begin{array}{l}\text { PLoS Public Library of Sci- } \\
\text { ence }\end{array}$ & $\begin{array}{l}\text { APC Association for Pro- } \\
\text { gressive Communication }\end{array}$ & SECOM \\
\hline Archive.org & CETID & SNAP \\
\hline LaQuadratureDuNet & AMJUMPRE.org.ec & $\begin{array}{l}\text { Diversas o todas las universida- } \\
\text { des del Ecuador. }\end{array}$ \\
\hline Mozilla Foundation & CDIecuador.org & $\begin{array}{l}\text { Ministerio de Telecomuncacio- } \\
\text { nes y de la sociedad de la Infor- } \\
\text { mación }\end{array}$ \\
\hline $\begin{array}{l}\text { Fundación Carisma (Colom- } \\
\text { bia) }\end{array}$ & E-ducate.org & Ministerio de Educación \\
\hline $\begin{array}{l}\text { Open Knowledge Foundation } \\
\text { http://okfn.org }\end{array}$ & Fedaeps.org & FLACSO \\
\hline $\begin{array}{l}\text { Xnet http://whois--X.net + } \\
\text { http://fcforum.net }\end{array}$ & Thoughtworks Ecuador & \\
\hline \multicolumn{3}{|l|}{$\begin{array}{l}\text { Open Source Ecology } \\
\text { http://opensourceecology.org }\end{array}$} \\
\hline Arduino Foundation & & \\
\hline
\end{tabular}

Quadro 1: Lista preliminar e incompleta de instituciones y agentes sociales nacionales e internacionales

\subsection{Herramientas digitales para la investigación y producción colaborativa y para la comunicación de propuestas y resultados}

A continuación explicamos la serie de herramientas que se encuentran ya disponibles para articular el proceso de investigación, producción y comunicación:

- $\quad$ EDX un curso edx sobre sociedad del conocimiento común y abierto . Sistema en producción en http://edx.alabs.org

- $\quad$ MediaWiki: es la plataforma wiki más completa y estable (la que utiliza Wikipeᄀdia), permite control de versiones de diferentes documentos, usuarios y fichas de usuario, subir documentos, etc. Vamos a añadir las siguientes propiedades. http://flok-society.iaen.edu.ec/wiki 
- Semantizado

- Control especial de versiones, idiomas y revisores o editores que den de alta nuevos cambios

○ 3 idiomas

- Software para hacer mapas que permita mapear instituciones, comunidades y asociaciones en Ecuador.

- Wordpress: [ya está operativo: http://flok-society.iaen.edu.ec]

○ Software Encuestas [puede que esto vaya en el wiki]

- Centrado en la comunicación: Portada con info organizada en 4 bloques y en cómo participar.

○ En 3 idiomas: Kichwa, castellano e inglés

- Incluye fichas de participantes colaboradores, integrando la información de usuarias del mediawiki.

- $\quad$ Mumble: servidor de vozip que permite crear salas para reuniones de trabajo. El cliente de Mumble está disponible para Windows, Mac y GNU/Linux: http://flok$\underline{\text { society.iean.edu.ec/mumble }}$

- $\quad$ Etherpad que permite la escritura en tiempo real para 16 personas al mismo tiempo: http://flok-society.iaen.edu.ec/pad

- $\quad$ Bibliografía compartida en Zotero para temas de conocimiento abierto, etc.

\subsection{Calendario}

El proceso de divide en 7 fases:

1. [FG] Fase de gestación: 1-30 de Agosto. Se diseña el proceso, se estudian nece-sidades de contratación, se van formando los equipos y se planifica la participa $\neg$ ción institucional.

2. [FI] Fase de incubación: 1-30 de Septiembre, primeras rondas de encuestas y despliegue de red de contactos, elaboración de los planes e infraestructura básica (técnica y humana)

3. [FD1] Fase de desarrollo 1: del 1 de Octubre al 25 de Noviembre. Lanzamiento testing y lanzamiento oficial, articulación primeros procesos de participación y mini-cumbre. 
4. [FD2] Fase de desarrollo 2: del 25 de Noviembre al 20 de Enero. Monitorizaᄀción del proceso de producción, reuniones de trabajo, y campañas de comunica $\neg$ ción.

5. [FC] Fase cumbre: del 20 de Enero al 15 de Marzo, preparación de la cumbre y desarrollo de la misma, elaboración de resultados y documentación.

6. [FPC] Fase post-cumbre: del 15 de Marzo al 30 de Abril. Elaboración, revisión y edición de los resultados.

7. [FIM] Fase de implementación y monitorización: el período es variable (posi-blemente de uno o dos años), cubre la fase de traducción de los resultados a iniาciativas legislativas específicas, así como la monitorización por parte de las sociędad civil de su implementación.

Detallamos a continuación un calendario con fechas, partes del proceso y resultados de los mismos.

Quadro 2: Las fases del proceso

\begin{tabular}{|c|c|c|}
\hline & Proceso & Resultados y Notas \\
\hline \multicolumn{3}{|l|}{ FASE 1: Gestación } \\
\hline 1-30 de Agosto 2013 & $\begin{array}{l}\text { Formación y desarrollo preliminar. } \\
\text { Reuniones con instituciones y } \\
\text { agentes sociales para presentar el } \\
\text { proyecto } \\
\text { Invitación de los actores más im- } \\
\text { portantes }\end{array}$ & $\begin{array}{l}\text {-Beta de la web } \\
\text {-Wiki con el documento madre. } \\
\text {-Desarrollo conceptual terminado } \\
\text {-Diseño (teórico) de la arquitectura } \\
\text { de participación. } \\
\text {-Identificación de actores y redes } \\
\text { participantes } \\
\text {-Definición en detalle de equipo de } \\
\text { trabajo y búsqueda de personal } \\
\text {-Documento de trabajo consensua- } \\
\text { do } \\
\text {-Repositorio bibliográfico de do- } \\
\text { cumentación existente tanto inter- } \\
\text { nacional como nacional sobre } \\
\text { sociedad del conocimiento abierto }\end{array}$ \\
\hline \multicolumn{3}{|l|}{ FASE 2: Incubación } \\
\hline 1-30 de Septiembre 2013 & $\begin{array}{l}\text { Despliegue y desarrollo de red de } \\
\text { apoyos, documentos base y siste- } \\
\text { mas informáticos } \\
\text { de participación. } \\
\text { Primera ronda de participación } \\
\text { vía encuestas. } \\
\text { Reuniones con instituciones } \\
\text { y agentes sociales para coordinar } \\
\text { la participación. }\end{array}$ & $\begin{array}{l}\text {-Mapa de red de apoyos y posibles } \\
\text { agentes participantes, nivel de } \\
\text { participación. } \\
\text { - Versiones beta de los documen- } \\
\text { tos base } \\
\text { - Diseño del sistema informático } \\
\text { de participación y producción } \\
\text { colectiva } \\
\text { - Contratación de personal para el } \\
\text { equipo de coordinación. } \\
\text { - Desarrollo de la encuesta por }\end{array}$ \\
\hline
\end{tabular}




\begin{tabular}{|c|c|c|}
\hline & $\begin{array}{l}\text { Invitación de los actores más } \\
\text { importantes y solicitud de } \\
\text { los informes. }\end{array}$ & $\begin{array}{l}\text { áreas } \\
\text { - Análisis de resultados de las } \\
\text { encuestas } \\
\text { - Integración de investigaciones } \\
\text { relevantes en repositorio y docu- } \\
\text { mentos resumen. }\end{array}$ \\
\hline \multicolumn{3}{|l|}{ FASE 3: Desarrollo 1} \\
\hline 5 de Octubre 2013 & $\begin{array}{l}\text { Lanzamiento testing de la } \\
\text { plataforma de participación } \\
\text { y campaña de comunicación } \\
\text { Terminar invitación de los } \\
\text { actores más importantes }\end{array}$ & $\begin{array}{l}\text { - Diseño de la campaña de comu- } \\
\text { nicación } \\
\text { web y social media para darle } \\
\text { publicidade al proceso-cumbre. } \\
\text { - Web con usuarios ya creados de } \\
\text { los agentes ya involucrados y con- } \\
\text { tactados } \\
\text { - Documentos base subidos para } \\
\text { invitar a } \\
\text { la participación. } \\
\text { - Listado de invitados especiales } \\
\text { confirmados ya disponible. }\end{array}$ \\
\hline 20 de Octubre 2013 & $\begin{array}{l}\text { Lanzamiento público de la } \\
\text { propuesta de participación y } \\
\text { comunicación }\end{array}$ & $\begin{array}{l}\text { - Web del proceso y puesta en } \\
\text { marcha de la arquitectura de la } \\
\text { participación } \\
\text { - Monitorización de los resultados } \\
\text { de participación, }\end{array}$ \\
\hline $\begin{array}{l}20 \text { de Octubre al } \\
20 \text { de Noviembre } 2013\end{array}$ & $\begin{array}{l}\text { Riego, cuidado, hibridación } \\
\text { analógico-digital e } \\
\text { integración del proceso de } \\
\text { participación. }\end{array}$ & $\begin{array}{l}\text { - Desarrollo de lista de participan- } \\
\text { tes implicados } \\
\text { - Informe de monitorización del } \\
\text { proceso } \\
\text { - Reuniones con actores locales y } \\
\text { traslado de resultados a la plata- } \\
\text { forma online. }\end{array}$ \\
\hline $\begin{array}{l}\text { 18-21 de } \\
\text { Noviembre } 2013\end{array}$ & $\begin{array}{l}\text { Encuentro preparativo con } \\
\text { actores principales e } \\
\text { instituciones }\end{array}$ & $\begin{array}{l}\text { - Resultados discusión } \\
\text { - Confirmada lista de invitados } \\
\text { especiales y } \\
\text { actores nacionales para el evento } \\
\text { final. }\end{array}$ \\
\hline \multicolumn{3}{|l|}{ FASE 4: Desarrollo 2} \\
\hline 27 de Noviembre 2013 & $\begin{array}{l}\text { Segundo lanzamiento } \\
\text { público orientado al } \\
\text { encuentro final. }\end{array}$ & $\begin{array}{l}\text { - Web y campaña de lanzamiento } \\
\text { anunciando invitados y resultados } \\
\text { preliminares } \\
\text { - Sistema de inscripción }\end{array}$ \\
\hline 20 de Enero 2014 & $\begin{array}{l}\text { Tercer lanzamiento } \\
\text { público antes del evento }\end{array}$ & $\begin{array}{l}\text { - Lista definitiva de participantes } \\
\text { - Programa de la cumbre, carteles, } \\
\text { etc. }\end{array}$ \\
\hline \multicolumn{3}{|l|}{ FASE 5: Cumbre } \\
\hline 1-15 de Febrero 2014 & Preparativos para la cumbre & $\begin{array}{l}\text { - Testeado de la red y sistemas } \\
\text { informáticos } \\
\text { - Revisión listado }\end{array}$ \\
\hline 17-23 de Febrero 2014 & Cumbre & $\begin{array}{l}\text { - } 10 \text { documentos programáticos en } \\
\text { versión alfa. } \\
\text { - dossier impacto mediático }\end{array}$ \\
\hline 24 de Febrero al & Elaboración de resultados & - 10 documentos revisados, tradu- \\
\hline
\end{tabular}




\begin{tabular}{|l|l|l|}
\hline 7 de Marzo 2014 & \multicolumn{1}{|c|}{$\begin{array}{l}\text { cidos, unificados en términos de } \\
\text { formato, etc. }\end{array}$} \\
\hline 15 de Marzo 2014 & $\begin{array}{l}\text { Publicación y difusión de } \\
\text { resultados abiertos }\end{array}$ & $\begin{array}{l}\text { - Publicación final de los resulta- } \\
\text { dos de la cumbre, versión Beta } \\
\text { - Campaña de difusión }\end{array}$ \\
\hline FASE 6: Post-cumbre & $\begin{array}{l}\text { - Publicación final de los resulta- } \\
\text { dos de la cumbre, versión Beta } \\
\text { abierta para su debate y revisión }\end{array}$ \\
\hline 15 de Marzo & $\begin{array}{l}\text { Publicación y difusión de } \\
\text { resultados abiertos }\end{array}$ & $\begin{array}{l}\text { - Documentos en versión estable y } \\
\text { cerrada. }\end{array}$ \\
\hline $\begin{array}{l}\text { 16 de Marzo al } \\
\text { 15 de Abril 2014 }\end{array}$ & $\begin{array}{l}\text { Fase final de discusión y } \\
\text { revisión de los documentos }\end{array}$ \\
\hline FASE 7: Implementación y monitorización & \\
\hline $\begin{array}{l}\text { Depende de la agenda institucional, cubre la fase de traducción de los resultados a } \\
\text { iniciativas legislativas específicas, así como la monitorización por parte de las sociedad civil } \\
\text { de su implementación. }\end{array}$
\end{tabular}

Quadro 2: Calendario con fechas, partes del proceso y resultados de los mismos.

\subsection{Recursos Humanos}

Los recursos humanos de un proyecto de esta magnitud son muy extensos, hablamos en total de unas 200-300 personas. Hemos dividido el tipo de participantes o integrantes implicados en tres grandes bloques: a) equipo coordinador (con varios subapartados), b) red de participantes, y c) red de editores y revisoras. A continuación se detalla la función y el tipo de equipos y contratos o formas de implicación de estas tres grandes categorías.

\section{Equipo coordinador}

Es fundamental contar con un equipo de desarrollo del evento que estaría compuesto por:

- Equipo de coordinación estratégica y político-institucional: 5-7 personas (asesores, prometeos, gerentes, ministros, etc.) que compartan una visión del proceso-cumbre y que coordinen su contenido en términos estratégicos y en términos de integración en el proyecto políticoinstitucional ecuatoriano.

- $\quad$ Equipo de diseño e integración: este es el equipo más importante, compuesto de unas 510 personas y las que tiene que haber al menos dos o tres dedicadas en exclusiva a monitorizar todo, al menos deben formar parte de este equipo un coordinador de cada uno de los siguientes equipos.

- Equipo de desarrollo técnico: encargado del diseños, implementación y mantenimiento 
de la arquitectura de producción colaborativa online, de la web, del sistema de coordinación digital durante la cumbre y los sistemas de control de versiones, etc.

- $\quad$ Equipo de producción: del evento cumbre y las reuniones preparativas previas, una especie de secretaría de producción.

- Equipo de comunicación y documentación: Encargado de difusión nacional e internacional, en prensa y televisión así como en la red, monitorización del impacto, creación de dossieres, documentación del proceso, etc.

- Coordinadores de área: muy importante que cada área tenga uno o varias coordinadoras que pueden ser de la red de editores y que mantengan una activa coordinación con el equipo de integración.

- Equipo de traducción: este equipo se encarga de hacer las traducciones inglés, kichwa, castellano para mantener la web y los documentos actualizados. La versión oficial será en castellano, siendo la de kichwa y la de inglés versiones de la misma. Las contribuciones en inglés o kichwa serán primero traducidas al caste-llano para ser o no aceptadas en el sistema de producción e investigación colaborativa. Esto requiere un equipo de traducción rápida.

Queremos habilitar 3 tipos de integrante del equipo coordinador en función de su dediᄀcación y del tipo de contrato:

1. Contratados a tiempo completo con un $100 \%$ de dedicación. A estas personas hay que contratarlas directamente desde su momento de incorporación hasta dos meses después de finalizar la cumbre, cuando todo el material esté ya operativa $\neg$ mente integrado en políticas de Estado. Aquí vamos a necesitar personas con perfiles de contratación muy diferentes, dependiendo de su CV y la responsabili $\neg$ dad y competencias requeridas para su tarea: hablamos de contratos desde $800 \$$ a $4000 \$$ al mes.

2. Un segundo grupo de integrantes ya tiene un contrato como servidor público: investigadores del IAEN, agentes sociales, ONGs, prometeos, equipos jurídicos, ase $\neg$ sores, equipos de trabajo ministeriales o de la SENESCYT, activistas, etc. Su dediᄀcación al proceso-cumbre puede variar de un $10 \%$ a un $50 \%$ de su trabajo coti-diano, pero esta dedicación y las funciones tienen que quedar bien definidas en una especie de "contrato" en el que asuman sus responsabilidades. Deberán per $\neg$ manecer siempre activas en su vinculación con los procesos de coordinación 
y producción con momentos de trabajo puntual más activos.

3. Un tercer grupo está compuesto por actores que no van a poder desplazarse a Quito permanentemente pero que quieren y puede participar puntualmente (visiᄀtas de un mes, antes, durante y después del evento) y que mantengan un segui $\neg$ miento telemático. Algunas de estas personas van a requerir contactos puntuales (quizás a modo de consultoría), otras puede que tengan ya algún tipo de contra-to como prometeos de uno o dos meses, o incluso puede que su misión dentro de sus organizaciones les permita tener una vinculación activa al proyecto sin coste añadido para Ecuador.

\section{Red de participantes}

En todos los casos nos referimos siempre a una proporción de al menos 2:1 de actores locales frente a extranjeros:

- Institucionales: asambleistas, rectores, funcionariado técnico, etc.

- $\quad$ Académicos e investigadoras

- Ciberactivistas y hackers

- $\quad$ Actores comunitarios de base

- $\quad$ Agentes económicos y de desarrollo: empresas, cooperativas, etc.

Red de editores y revisoras

Esta figura está por determinar pero el proceso de participación debe estar coordinado activamente por una serie de editores "expertos" que vayan a aceptar las modificaciones. Este equipo estará en gran medida compuesto por una selección de actores de la red de participantes. Esta red debe formarse pronto para que funcione también como una especie de consejo consultivo en varios momentos del proceso.

Plan de contratación

En las primeras dos fases de gestación e incubación (hasta el 1 de Octubre) necesitamos urgentemente a una serie de personas, pueden ser contratadas o que ya tengan un puesto de servidor público y se encuentren disponibles para comenzar a trabajar en el even-to, con reuniones 
diarias y formación continua. Después tendrán que completarse los equipos. Dividimos las necesidades de contratación entre contrataciones urgente (de inmediata aplicación a ser posible) y contrataciones importantes (medio plazo) necesario.

\section{Urgente}

- 1 Diseñador/a: tiene que diseñar imagen corporativa y adaptarla a la web, presentaciones, etc. Tiene que tener un perfil mixto de diseño corporativo y diseño web.

- 1 Desarrollador/a web: desarrollo web con php, css, especialista en MediaWiki y Wordpress, web semántica, multi-idioma y a poder ser visualización de datos.

- 1 Asistente de pre-producción: encargada de contratación de personal, gestión de calendarios, contactos, gestiones de secretariado varias. Se requiere eficiencia, nivel medio-alto de inglés, y conocimiento de modelos de contratación y gestión dentro de las instituciones públicas. [podría ser la misma persona que asistente de coordinación si cumple requisitos, ver más abajo]

- 1 Coordinador/a de Comunicación: esta persona tendrá que coordinar un equipo de comunicación que irá creciendo durante el desarrollo del proyecto. Tiene que tener un perfil avanzado en comunicación tanto en castellano como en inglés, con gran manejo de los social media, a poder ser con experiencia en redes sociales internacionales como Reddit, conocedor/a de las comunidades del ciberespacio (boing-boing, wikipedia foundation, EFF, creative commons, etc.).

- $\quad$ Equipo de traducción: externalizado para servicios puntuales de traducción urgente (más tarde hará falta un equipo permanente de traductores, sobre todo para la cumbre).

Importante pero no urgente

- 1 Asistente de Coordinación: una persona con una capacitación muy alta, perᄀfil medio político, medio producción, medio facilitación y coordinación de gran $\neg$ des eventos. Tiene que tener conocimientos avanzados de TIC, sociedad red, etc. a poder ser experimentada/o en entornos de trabajo GNU/Linux: MediaWiki, Wordpress, OpenOffice, Zimbra, etc.

- 1 Administrador/a de sistemas: experto en servidores GNU/Linux (preferible $\neg$ mente Debian/Ubuntu) con experiencia en NGINX, MySQL, postfix, etc. 


\section{REFERÊNCIAS}

ALTMAN, David. Bringing direct democracy back in: toward a three-dimensional measure of democracy. Democratization, Londres, v. 20, n. 4, p.615-641, abr. 2012. Disponível em: <http://dx.doi.org/10.1080/13510347.2012.659020>. Acesso em: 18 jul. 2014.

BAUWENS, Michel. The basic orientation of P2P theory towards societal reform: transforming civil society, the private and the state. 2011. Disponível em:

$<$ http://blog.p2pfoundation.net/the-basic-orientation-of-p2p-theory-towards-societal-reformtransforming-civil-society-the-private-and-the-state/2011/07/12>. Acesso em: 18 jul. 2014.

BOLLIER, David. The city of linz pioneers a regional information commons. 2011. E-book. Disponível em: <http://bollier.org/city-linz-pioneers-regional-information-commons>. Acesso em: 21 jul. 2014.

BOLLIER, David; HELFRICH, Silke. Introduction: the commons as a transformative vision. 2013. Disponível em: <http://wealthofthecommons.org/essay/introduction-commonstransformative-vision>. Acesso em: 21 jul. 2014.

D'ARISTOTE au web de demain: i'intelligence collective selon Pierre Lévy. 2013. Disponível em: <http://unblogdesblogs.wordpress.com/2013/07/02/daristote-au-web-de-demainlintelligence-collective-selon-pierre-levy/>. Acesso em: 21 jul. 2014.

Fix my Street. Disponível em: <http://www.fixmystreet.com/>. Acesso em: 21 jul. 2014.

FUNDAÇÃO GETÚLIO VARGAS (Rio de Janeiro). Últimos dias para o envio de Ideias para o Rio+. FGV Direito Rio. Rio de Janeiro. 14 out. 2013. Disponível em:

<http://direitorio.fgv.br/ultimos_dias_rio>. Acesso em: 21 jul. 2014.

HARDT, Michael; NEGRI, Antonio. Declaration. 2012. E-book. Disponível em: <http://www.amazon.com.br/Declaration-Michael-Hardt-ebook/dp/B00816QAFY>. Acesso em: 21 jul. 2014.

HELFRICH, Silke. The Commons: year one of the global commons movement. 2011. Disponível em: <http://commonsblog.wordpress.com/2011/01/29/the-commons-year-one-of-theglobal-commons-movement/>. Acesso em: 21 jul. 2014.

HEYLIGHEN, Francis. Return to Eden? promises and perils on the road to a global. 2013. Disponível em: <http://pespmc1.vub.ac.be/Papers/BrinkofSingularity.pdf>. Acesso em: 21 jul. 2014. 
Human Devolepment Reports. Disponível em: <http://hdr.undp.org/en/statistics/mpi/>. Acesso em: 21 jul. 2014.

LÉVY, Pierre. L'intelligence collective: pour une anthropologie du cyberespace. Paris: La Découverte, 2004. 252 p. (Essais n.27)

MATTEI, Ugo. First thoughts for a phenomenology of the commons. 2013. Disponível em: <http://wealthofthecommons.org/essay/first-thoughts-phenomenology-commons>. Acesso em: 21 jul. 2014.

MATTEI, Ugo. L'Etat, le marché et quelques questions préliminaires à propos du commun. 2011. Disponível em: <http://works.bepress.com/cgi/viewcontent.cgi?article= 1040\&context=ugo_mattei>. Acesso em: 21 jul. 2014

MERETZ, Stefan. The structural communality of the commons. 2013. Disponível em: <http://wealthofthecommons.org/essay/structural-communality-commons>. Acesso em: 21 jul. 2014.

MILUN, Kathryn. Wrapping our legal mnds around the global commons: res nullius, res communis, and res divini juris, On the Commons. 2007. Disponível em:

$<$ http://onthecommons.org/wrapping-our-legal-minds-around-global-commons-res-nullius-rescommunis-and-res-divini-juris>. Acesso em: 21 jul. 2014.

Open Commons. Disponível em: <http://opencommons.public1.linz.at/>. Acesso em 21 jul. 2014.

P2P Fundation. Disponível em: <http://p2pfoundation.net>. Acesso em 21 jul. 2014. 